\title{
MODELOS DE DUPLA CAMADA DIFUSA DE GOUY-CHAPMAN E STERN APLICADOS A LATOSSOLOS ÁCRICOS PAULISTAS ${ }^{1}$
}

\author{
L.R.F. ALLEONI \\ Seção de Algodäo/IAC, C.P. 28 - CEP: 13020-902 - Campinas,SP \\ O.A. de CAMARGO ${ }^{2}$ \\ Seção de Pedologia/IAC, C.P. 28 - CEP: 13020-902 - Campinas,SP.
}

RESUMO: Modelos teoricos da dupla camada difusa de Gouy-Chapman e de Stern foram aplicados a um Iatossolo variação Una ácrico, de Guaíra e a dois latossolos roxos ácricos, localizados em Ribeirão Preto e Guaíra, cidades ao norte do Estado de São Paulo, onde solos com caráter ácrico ocupam vasta área nas quais são cultivadas várias culturas de interesse econômico. Na concentração mais elevada do eletrólito suporte (KCl $0,1 \mathrm{~N}$ ), os dados obtidos em laboratório adaptaram-se melhor ao modelo de Stern. Entretanto, nas menores concentrações $(\mathrm{KCl} 0,01$ e $0,001 \mathrm{~N})$, correspondentes a valores mais comuns encontrados em solos altamente intemperizados, o modelo de Gouy-Chapman representou melhor a variaçāo da densidade superficial de carga com o potencial elétrico das amostras estudadas.

Descritores: solos ácricos, dupla camada difusa, modelos de Gouy-Chapman e Stern.

\section{GOUY-CHAPMAN AND STERN DIFFUSE DOUBLE LAYER MODELS APPLIED TO ACRIC OXISOLS FROM THE STATE SÃO PAULO - BRAZIL}

ARSTRACT: Theoretical models of Gouy-Chapman and Stern, referred to diffuse double layer, were applied to three acric Oxisols, two named dusky red latosol and one Una variant latosol, from the São Paulo State - Brazil. Samples were collected in Ribeirão Preto and Guaira, regions of large occurrence of these soils, supporting many economic crops. In the highest concentration of the electrolyte ( $\mathrm{KCl} 0.1 \mathrm{~N})$, the obtained values were better related to the Stern model. On the other hand, in more dilluted solutions (KCl 0.01 and $0.001 \mathrm{~N}$ ), corresponding to more usual ionic strenght of highly weathered soils, the variation of surface charge density of the samples with electric potential was in better agreement with the Gouy-Chapman theory.

Key wonds: acric oxisols, diffuse double layer, Gouy-Chapman and Stern models

\section{INTRODUÇÃO}

A superfície dos colóides do solo possui carga elétrica líquida predominantemente negativa. Íons de carga positiva, que estão normalmente dissociados na solução do solo, tendem a permanecer na vizinhança desta superfície, atraídos que são pelo campo elétrico formado (BOLT, 1978). As forças de difusão tendem a trazer estes cátions de volta à solução em equilibrio, onde sua concentração é menor. Com a ação concomitante destas duas forças opostas, uma distribuição espacial de cátions numa "camada difusa" é estabe- lecida, na qual a concentração deles aumenta em direção à superfície, partindo de um valor igual àquele na solução para um valor maior, determinado, principalmente, pela magnitude da carga superficial (UEHARA \& GILLMAN, 1981).

\section{A teoria de Gouy-Chapman}

Esta teoria descreve a distribuição de íons próximos à superfície carregada dos colóides, relacionando a carga com o potencial elétrico na superfície. Assumindo um eletrólito simétrico, tem-se a seguinte equação (RAIJ, 1971):

\footnotetext{
1 Parte da Dissertação de Mestrado apresentada pelo primeiro autor à Escola Superior de Agricultura "Luiz de Queiroz"/USP, para obtenção do título de Mestre em Solos e Nutrição de Plantas.

${ }^{2}$ Bolsista do CNPq.
} 
$\sigma=(2 \eta \in k T / \pi)^{1 / 2} \operatorname{senh}\left(z e \psi_{0} / 2 k T\right)$

onde: $\sigma=$ densidade de carga superficial $\left(\mathrm{meq} / \mathrm{cm}^{2}\right) ; \eta=$ concentração do eletrólito $(\mathrm{mol} / \mathrm{l})$ ; $\epsilon=$ constante dielétrica do meio; $k=$ constante de Boltzmann; $\mathbf{T}=$ temperatura absoluta ( $\left.{ }^{\circ} \mathrm{K}\right) ; \pi=$ 3,1416; $z=$ valência do contra-íon; $e=$ carga do elétron (coulomb) e $\psi_{0}=$ potencial elétrico na superfície $(\mathrm{mV})$.

Num sistema de cargas variáveis, o potencial da superfície $\epsilon$ controlado pela adsorção de íons determinadores de potencial, a qual por sua vez depende da atividade destes íons na solução (UEHARA \& GILLMAN, 1981). O valor de $\psi_{0}$, em $\mathrm{mV}, \epsilon$ obtido usando-se a equação de Nernst, simplificada por RAIJ \& PEECH (1972):

$\psi_{0}=59,1(\mathrm{PESN}-\mathrm{pH})$

onde: PESN = ponto de efeito salino nulo, termo preferido em relação a PCZ (ponto de carga zero), de acordo com proposição de ALLEONI \& CAMARGO (1993), já que o método utilizado para sua obtenção foi a titulação potenciométrica das amostras em diferentes concentrações de eletrólito, conforme CAMARGO et al. (1986).

A aplicação das equações 1 e $2 \mathrm{em}$ colóides com carga permanente ou constante e com carga variável ou interface reversível (RAIJ \& PEECH, 1972) permite algumas observações importantes. Nos componentes com carga superficial constante, tais como solos pouco desenvolvidos, um aumento na concentração do eletrólito, na valência do íon ou na constante dielétrica do meio terá que ser necessariamente seguido de um decréscimo no potencial elétrico da superfície, de modo a manter a densidade de carga superficial constante. Este fenômeno É acompanhado por uma redução na espessura ou, como afirmam UEHARA \& GILLMAN (1981), por uma compressão da dupla camada elétrica. Para colóides com interface reversível, o potencial na superfície num dado $\mathrm{pH}$, que é obtido a partir da equação 2 , $\varepsilon$ independente da concentração do eletrólito. Por essa razão, ocorre aumento na densidade de carga quando, por exemplo, a concentração do eletrólito aumenta (BELL \& GILLMAN, 1978).

A equação de Gouy-Chapman, embora muito utilizada, não é adequada para situações em que a concentração salina $\epsilon$ alta (RAIJ, 1971) ou quando são utilizados valores moderadamente altos de potencial (UEHARA \& GILLMAN, 1981). Isto ocorre devido ao fato de os íons serem considerados cargas pontuais e poderem aproximar-se indefinidamente da superficie carregada.

\section{A teoria de Stern}

Stern introduziu correções no modelo de Gouy-Chapman, principalmente levando em conta o tamanho finito dos íons (RAIJ, 1986), ou seja, eles não poderiam se aproximar da superfície além de uma certa distância, alguns poucos nanômetros, segundo UEHARA \& GILLMAN (1981). Nesta teoria, assume-se que existem duas camadas: uma mais próxima à superfície $\left(\sigma_{1}\right)$, chamada de camada de Stern, representada pelos íons adsorvidos, e outra composta pelos outros ions, formando uma camada difusa $\left(\sigma_{2}\right)$. A parte da dupla camada entre - plano no qual a superfície do colóide está localizada e o plano de Stern, que passa pelos contra-íons, $\varepsilon$ considerada um condensador molecular, no qual o potencial $\psi_{0}$ decresce linearmente com a distância da superfície da partícula para um valor $\psi_{0}$ no plano de Stern (SIQUEIRA, 1985). A densidade de cargas nas camadas de Stern é dada por:

$\sigma_{1}=\mathrm{N}_{1} . z . e / 1+\left(\mathrm{N}_{A} \rho / \mathrm{Mn}\right) \exp \left[-\left(\mathrm{z} . \mathrm{e} . \psi_{\delta}+\phi\right) / \mathrm{kT}\right]$.

onde: $\mathbf{N}_{1}=$ número disponível de sítios, por $\mathrm{cm}^{2}$, para adsorção de ions; $\mathrm{N}_{\mathrm{A}}=$ número de Avogadro; $M=$ massa molecular do solvente $=18 \mathrm{~g} / \mathrm{mol}$ para água; $\mathfrak{n}=$ concentração de íons $(\mathbf{n}=$ moles $/$ litro $x$ $n^{0}$ de Avogadro); $\rho=$ densidade do solvente; $\psi_{0}=$ potencial elétrico no plano de Stern e $\phi=$ potencial de adsorção específica do contra-íon pelo colóide.

A densidade total de carga é dada por:

$\sigma=\sigma_{1}+\sigma_{2}$

A densidade de carga superficial é também obtida pela equação para o condensador molecular (RAIJ, 1971):

$\sigma=\left(\epsilon^{\prime} / 4 \pi \delta\right) \times\left(\psi_{0}-\psi_{\delta}\right)$

onde $: \epsilon^{\prime}=$ "constante" dielétrica média na camada de Stern e $\delta=$ espessura da camada de Stern em nm.

O objetivo deste trabalho é verificar a adequação dos modelos de Gouy-Chapman e de Stern a três latossolos ácricos do norte paulista. 


\section{MATERIAL E MÉTODOS}

Foram utilizadas amostras superficiais e subsuperficiais de dois latossolos roxos ácricos (LRác-1 e LRác-2, localizados em Ribeirão Preto e Guaíra, respectivamente) e um latossolo variação Una ácrico (LUác), de Guaíra. Ambas as cidades localizam-se ao norte do estado de São Paulo e sustentam uma agricultura altamente tecnificada. Para que fossem aplicados os modelos de Gouy-Chapman e de Stern, as equações 1, 2, 3, 4 e 5 foram simplificadas de acordo com RAIJ (1971), dando origem a novas equações:

$$
\begin{aligned}
& \sigma_{2}=1,218 \cdot 10^{-7} \mathrm{c}^{1 / 2} \operatorname{senh}\left(0,0195 \cdot \mathrm{z} \cdot \psi_{\delta}\right) \ldots . .(7) \\
& \sigma_{1}=16,6 \cdot 10^{-7} \cdot \mathrm{z} / 1+(55,6 / \mathrm{c}) \exp \left(-0,039 \cdot \mathrm{z} \cdot \psi_{\delta}\right) \cdot(8) \\
& \psi_{0}-\psi_{\delta}=181,7 \cdot 10^{6} . \delta . \sigma \ldots \ldots \ldots \ldots \ldots \ldots(9)
\end{aligned}
$$

Os potenciais elétricos, $\psi_{0}$ e $\psi_{\delta}$, são dados em $\mathrm{mV} ; \sigma_{1}, \sigma_{2}$ e $\sigma \mathrm{em} \mathrm{meq} / \mathrm{cm}^{2} ;$ c é a concentração molar do eletrólito simétrico na solução em equilíbrio e $\delta$ aparece em nanômetros. Considerou-se a ausência de adsorção específica, $\operatorname{assim} \phi=0$.

A carga superficial líquida pela teoria de Gouy-Chapman foi determinada pela equação 7 , após substituir $\psi_{\delta}$ por $\psi_{\mathrm{o}}$. Cálculos para utilização do modelo de Stern foram feitos com as equações $4,7,8$ e 9.

Para obtenção de $\sigma_{2}, \sigma_{1}, \sigma$ e $\psi_{\delta}$ foi utilizada a solução numérica de RAIJ (1971), assumindo-se valores diferentes para $\psi_{\delta}$, obtendo-se $\sigma_{2}, \sigma_{1}, \sigma$ e $\psi_{0}$ nesta ordem. Na TABELA 1 aparecem os valores assumidos de $\psi_{\delta}$ e os demais parâmetros obtidos posteriormente. Tomando-se, como exemplo, $\psi_{\delta}=5 \mathrm{mV} ; \mathrm{c}=0,01 \mathrm{M} ; \mathrm{z}=1$ (eletrólito 1:1); $\delta=0,1 \mathrm{~nm}$, os demais atributos foram assim calculados: inicialmente foi determinada a carga na dupla camada difusa $\left(\sigma_{2}\right)$, através da equação (7). A seguir, obteve-se a carga na camada de Stern $\left(\sigma_{1}\right)$, utilizando-se a equação (8). Somando-se $\sigma_{2}$ e $\sigma_{1}$, obteve-se a carga superficial total $(\sigma)$. Finalmente, com a densidade de carga conhecida, obteve-se o potencial elétrico na dupla camada difusa $\left(\psi_{0}\right)$, com auxílio da equação (9). Estes valores de $\sigma_{2}, \sigma_{1}, \sigma$ e $\psi_{\mathrm{o}}(0,12$; 0,$04 ; 0,16$ e 8) estão destacados na TABELA 1.

Nas Figuras 1, 2 e 3 plotaram-se os valores de carga elétrica superficial em função do potencial elétrico, sendo apresentados também os pHs nos quais estes pontos foram obtidos. As curvas indicadas por Gouy-Chapman representam nestas figuras a relação entre $\sigma_{2}$ e $\psi_{\delta}$, e as curvas indicadas por Stern representam a relação entre $\sigma$ e $\psi_{\mathrm{o}}$. A carga elétrica líquida superficial, em meq. $10^{-8} / \mathrm{cm}^{2}$, foi obtida após a divisão da carga elétrica líquida (em meq/100g) pela superfície específica $\left(\mathrm{em} \mathrm{m}^{2} / \mathrm{g}\right)$. Segundo LAVERDIËRE \& WEAVER (1977), a expressão dos valores de carga em relação à superfície específica fornece melhores comparações entre os solos, daí ter-se optado por esta unidade. Para a construção das figuras, foram considerados apenas os pontos entre $\mathrm{pH} 4,0$ e 7,5, faixa que engloba a grande maioria dos solos tropicais.

\section{RESULTADOS E DISCUSSÃO}

Observa-se que, nas três concentrações de $\mathrm{KCl}$, valores positivos de potencial e de carga elétrica líquida somente apareceram nas amostras do horizonte $B$, onde 0 efeito da matéria orgânica, no sentido de abaixar o ponto de efeito salino nulo e gerar cargas negativas, foi menor. Isto pode ser comprovado analisando-se a Figura $2(\mathrm{KCl} 0,01 \mathrm{~N})$ onde, para um mesmo valor de potencial elétrico, por exemplo $-65 \mathrm{mV}$, a carga líquida no horizonte A foi maior, próxima de $-3,2 \mathrm{meq} .10^{-8} / \mathrm{cm}^{2}$, contra $-1,4 \mathrm{meq} \cdot 10^{-8} / \mathrm{cm}^{2}$ do horizonte $\mathrm{B}$, mesmo tendo um pH menor: 4,5 contra 6,8 .

Para todas as curvas, se forem tomados isoladamente os pontos da camada superficial ou da subsuperficial, nota-se que os modelos descreveram bem a variação da densidade de carga líquida com o potencial elétrico do meio. Valores cada vez maiores, no lado negativo, foram observados à medida que o $\mathrm{pH}$ foi aumentando. Isto significa que quanto mais alto o $\mathrm{pH}$, mais distantes seus valores ficaram em relação ao PESN, acarretando assim um potencial maior (equação 2). Como conseqüência, o valor da carga elétrica negativa aumentou (equações 1 e 3 ).

Observou-se, também, que para ser atingido um mesmo valor de carga líquida (por exemplo $-3,0$ meq. $10^{-8} / \mathrm{cm}^{2}$ ), foi necessário um valor menor de $\psi_{0}$ na maior concentração de $\mathrm{KCl}$ $0,1 \mathrm{~N}(-37 \mathrm{mV}$ no modelo de Gouy-Chapman e $-85 \mathrm{mV}$ no modelo de Stern), um valor intermediário em $\mathrm{KCl} \quad 0,01 \mathrm{~N} \quad(-85 \mathrm{mV}$ para Gouy-Chapman e $-135 \mathrm{mV}$ para Stern) e um valor mais alto (em módulo) em $\mathrm{KCl} 0,001 \mathrm{~N}(-140 \mathrm{mV}$ para Gouy-Chapman e $-180 \mathrm{mV}$ para Stern). Devido ao fato de o potencial ser dependente do $\mathrm{pH}$ do meio (equação 2), pode-se concluir que uma 
TABELA 1. Valores calculados de carga na camada de Stern $\left(\sigma_{1}\right)$, na camada difusa $\left(\sigma_{2}\right)$, a carga elétrica líquida $(\sigma)$ e o potencial elétrico na dupla camada $\left(\psi_{0}\right)$, para valores selecionados de potencial de Stern $\left(\psi_{s}\right)$. Considerou-se um eletrolito simétrico $1: 1$ e $\delta=0,1 \mathrm{~nm}$. A carga é dada em $10^{8}$ $\mathrm{meq} / \mathrm{cm}^{2}$, e o potencial $\mathrm{em} \mathrm{mV}^{(1)}$.

\begin{tabular}{|c|c|c|c|c|c|c|c|}
\hline \multirow{2}{*}{ Concentração } & \multicolumn{7}{|c|}{ Potencial de Stem, $\psi_{\delta}$} \\
\hline & & 5 & 10 & 20 & 50 & 80 & 150 \\
\hline \multirow{4}{*}{$0,1 \mathrm{~N}$} & $\sigma_{1}$ & 0,36 & 0,44 & 0,65 & 2,07 & & \\
\hline & $\sigma_{2}$ & 0,38 & 0,76 & 1,54 & 4,38 & & \\
\hline & $\sigma$ & 0,74 & 1,20 & 2,19 & 6,45 & & \\
\hline & $\psi_{0}$ & 18 & 32 & 60 & 167 & & \\
\hline \multirow{4}{*}{$0,01 \mathrm{~N}$} & $\sigma_{1}$ & 0,04 & 0,04 & 0,07 & 0,21 & 0,67 & \\
\hline & $\sigma_{2}$ & 0,12 & 0,24 & 0,49 & 1,38 & 2,77 & \\
\hline & $\sigma$ & 0,16 & 0,28 & 0,56 & 1,59 & 3,44 & \\
\hline & $\psi_{0}$ & 8 & 15 & 30 & 78 & 143 & \\
\hline \multirow{4}{*}{$0,001 N$} & $\sigma_{1}$ & 0,00 & 0,00 & 0,01 & 0,02 & 0,07 & 1,04 \\
\hline & $\sigma_{2}$ & 0,04 & 0,08 & 0,15 & 0,44 & 0,88 & 3,58 \\
\hline & $\sigma$ & 0,04 & 0,08 & 0,16 & 0,46 & 0,95 & 4,62 \\
\hline & $\psi_{0}$ & 6 & 11 & 23 & 58 & 97 & 234 \\
\hline
\end{tabular}

(1) adaptado de RAIJ (1971).

variação neste $\mathrm{pH}$ tem efeito mais marcante na carga líquida de um solo quanto maior for sua força iônica, o que pode ocorrer, por exemplo, quando um adubo $\epsilon$ aplicado no solo. No caso dos latossolos ácricos, esta afirmativa é bastante importante, devido a baixíssima capacidade de retenção de cátions que estes solos possuem.

Como a força iônica da solução do solo, segundo BELL \& GILLMAN (1978) e BLACK \& CAMPBELL (1982), dificilmente supera 0,005 sendo comuns valores em torno de 0,002 - parece mais adequado comparar os dados obtidos de latossolos ácricos com as concentrações 0,01 e $0,001 \mathrm{~N}$ de $\mathrm{KCl}$ (Figuras 2 e 3).

$\mathrm{Na}$ maior concentração de $\mathrm{KCl}(0,1 \mathrm{~N})$ houve uma tendência de os pontos ajustarem-se melhor à curva de Stern, principalmente os relativos às camadas subsuperficiais, sendo que à medida que a concentração do sal foi diminuindo, os pontos foram se aproximando mais do modelo de Gouy-Chapman. Entretanto, é importante lembrar que para valores de potencial acima de
$100 \mathrm{mV}$, o modelo de Gouy-Chapman prevê densidades de carga que são fisicamente impossíveis de ocorrer (OLPHEN, 1963).

LAVERDIËRE \& WEAVER (1977) obtiveram resultados semelhantes com amostras de seis espodossolos dos Estados Unidos e do Canadá, sendo que apenas uma amostra seguiu o modelo de Stern, tendo as demais amostras apresentado densidades superficiais de carga, num dado potencial, superiores às que o modelo previa. Nesse trabalho, os autores afirmam que em solos com alto teor de hidróxidos metálicos, como é o caso dos latossolos ácricos, a variação na composição e no grau de cristalinidade dificultam, em parte, a adequação exata destes modelos teóricos. Além disso, RAIJ \& PEECH (1972) complementam que o pior ajuste dos pontos ao modelo de Stern na concentração de $\mathrm{KCl} 0,001 \mathrm{~N}$ é esperado, já que na determinação das cargas por titulação potenciométrica assume-se a auséncia de $\mathrm{H}^{+}$como contra-íon na dupla camada, condição difícil de ser encontrada em baixas concentrações de eletrolito. 


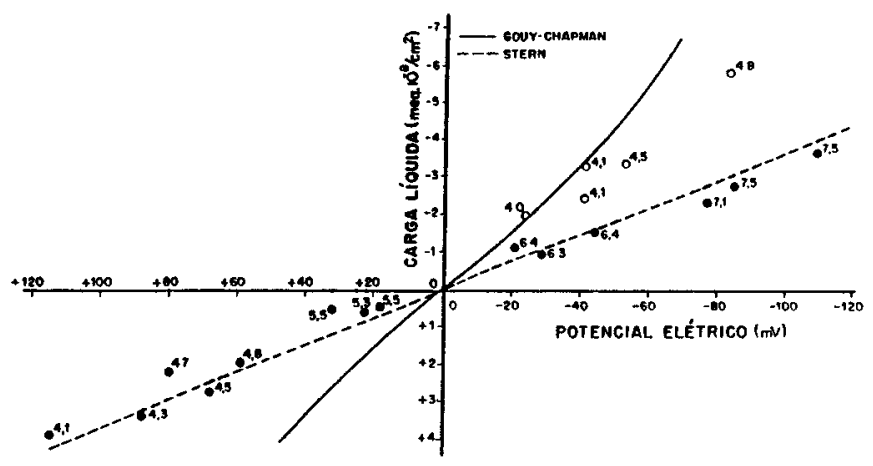

Figura 1. Potencial elétrico e carga líquida das amostras, numa solução de $\mathrm{KCl} 0,1 \mathrm{~N}$, e modelos teóricos de Gouy-Chapman e Stern. Os números que aparecem junto aos pontos mostram o pH da solução (o = horizonte superficial e $\bullet=$ horizonte subsuperficial).

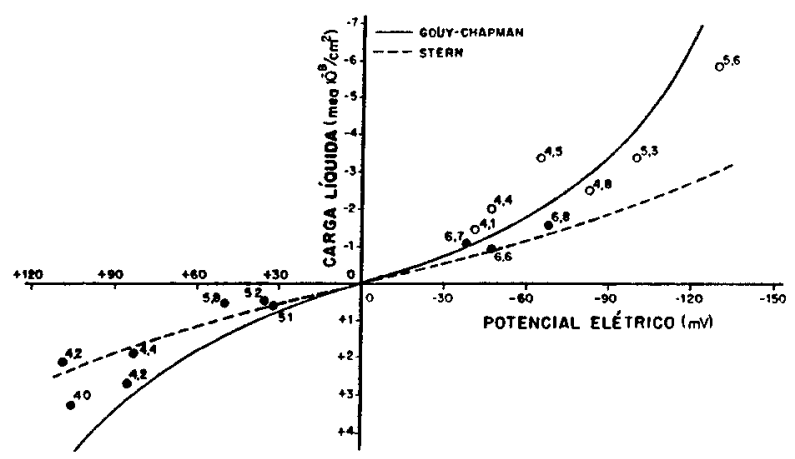

Figura 2. Potencial elétrico e carga líquida das amostras, numa solução de $\mathrm{KCl} 0,01 \mathrm{~N}$, e modelos teóricos de Gouy-Chapman e Stern. Os números que aparecem junto aos pontos mostram o pH da solução (o $=$ horizonte superficial $\mathrm{e} \bullet=$ horizonte subsuperficial).

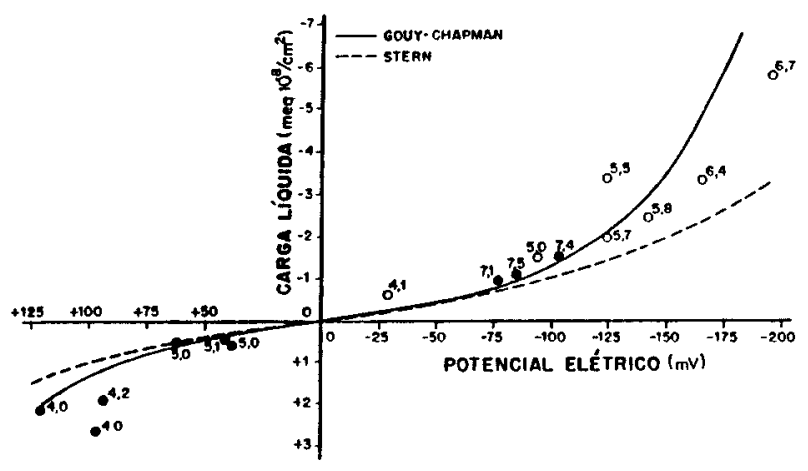

Figura 3. Potencial elétrico e carga líquida das amostras, numa solução de $\mathrm{KCl} 0,001 \mathrm{~N}$, e modelos teóricos de Gouy-Chapman e Stern. Os números que aparecem junto aos pontos mostram o pH da solução (o $=$ horizonte superficial e $\bullet=$ horizonte subsuperficial). 


\section{CONCLUSÕES}

1. Houve boa correlação entre os modelos teóricos e os valores obtidos em laboratório.

2. Nas concentrações de eletrólito mais próximas dos valores usuais de força iônica de solos altamente intemperizados, o modelo de Gouy-Chapman representou melhor a distribuição da densidade superficial de carga em função do potencial elétrico das amostras estudadas.

\section{REFERENCIAS BIBLIOGRÁFICAS}

ALleoni, L.R.F.; Camargo, O.A. Ponto de efeito salino nulo: proposição de nomenclatura. Boletim Informativo da Sociedade Brasileira de Ciencia do Solo, Campinas, v.18, p.5-11, jan./abr. 1993.

BELL, L.C.; GILLMAN, G.P. Surface charge characteristics and soil solution composition of highly weathered soils. In: ANDREW, C.S.; KAMPRATH, E.J., (ed). Mineral nutrition of legumes in tropical and subtropical soils. Melbourne: CSIRO, 1978. cap.2, p.37-57.

BLACK, A.S.; CAMPBELL, A.S. Ionic strength of soil solution and its effect on charge properties of some New Zealand soils. Journal of Soil Science, London, v.33, p.249-262, 1982.

BOLT, G.H. Surface interaction between the soil solid phase and the soil solution. In: BOLT, G.H.; BRUGGENWERT, M.G.M., (ed). Soil chemistry Part A. Basic elements. 2.ed. Amsterdam: Elsevier, 1978. cap.3, p.43-53.

CAMARGO, O.A.; MONIZ, A.C.; JORGE, J.A.; VALADARES, J.M.A.S. Métodos de análise química, mineralógica e física de solos do Instituto Agronômico de Campinas. Campinas: Instituto Agronômico, 1986. 94p. (IAC. Boletim Técnico, 106).
LAVERDIÈRE, M.R.; WEAVER, R.M. Charge characteristics of spodic horizons. Soil Science Society of America Journal, Madison, v.41, p.505-510, 1977.

OLPHEN, H. van. An introduction to clay colloid chemistry. New York: Interscience, 1963.340p.

RAIJ, B. van. Electrochemical properties of some Brazilian soils. Ithaca, 1971. 144p. Tese (Doutoramento) - Comell University.

RAI, B. van. Propriedades eletroquímicas de solos. In: SIMPósIo AVANÇADO DE QUÚMICA E FERTILIDADE DO SOLO, 1., 1986, Piracicaba. Anais... Campinas: Fundação Cargill, 1986. p.9-41.

RAIJ, B. van ; PEECH, M. Electrochemical properties of some Oxisols and Alfisols of the tropics. Soil Science Society of America Proceedings, Madison, v.36, p.587-593, 1972.

SIQUEIRA, C. Eletroquímica de solos tropicais de carga variavel: efeitos da matéria orgánica. Itaguaí, 1985. 113p. Tese (Doutoramento) - Universidade Federal Rural do Rio de Janeiro.

UEHARA, G. ; GLLMAN, G. The mineralogy, chemistry and physics of tropical soils with variable charge clays. Boulder: Westview, 1981. 170p.

Enviado para publicação em 02.03 .94

Aceito para publicaçăo em 20.04.94 